\title{
Effects of Micro Vibration Therapy Nursing Care on Muscle Hardness and Skin Blood Flow: A Pre/Post Group Comparison Study
}

\author{
Mari Azuma $^{1,2}$ (1), Chiharu Akazawa ${ }^{3}$ \\ ${ }^{1}$ Doctoral Course, Graduate School of Nursing, Osaka Medical and Pharmaceutical University, Osaka, Japan \\ ${ }^{2}$ Tenri Health Care University, Tenri, Japan \\ ${ }^{3}$ Osaka Medical and Pharmaceutical University, Osaka, Japan \\ Email: azuma.m@tenriyorozu-u.ac.jp
}

How to cite this paper: Azuma, M. and Akazawa, C. (2021) Effects of Micro Vibration Therapy Nursing Care on Muscle Hardness and Skin Blood Flow: A Pre/Post Group Comparison Study. Health, 13, 1511-1529. https://doi.org/10.4236/health.2021.1312108

Received: December 4, 2021

Accepted: December 27, 2021

Published: December 30, 2021

Copyright $\odot 2021$ by author(s) and Scientific Research Publishing Inc. This work is licensed under the Creative Commons Attribution International License (CC BY 4.0).

http://creativecommons.org/licenses/by/4.0/ (c) (i) Open Access

\begin{abstract}
Purpose: The purpose of this study was to investigate the effects of Micro Vibrational therapy (MVT) on muscle stiffness and blood flow in the skin before and after Micro Vibrational therapy in healthy subjects in order to scientifically verify the effects of MVT. Methods: Micro Vibrational therapy is nurse care use in Japan. It was performed on the backs of 30 subjects (8 males and 22 females) in their $20 \mathrm{~s}$ to $50 \mathrm{~s}$ according to the eligibility criteria. The resting state before implementation was set as the baseline for the control group, and after 30 seconds of MVT was set as the intervention group. The effects of the MVT were statistically analyzed by these factors and subjective sensation by Visual Analog Scale. Results: The muscle hardness of the area where the MVT was applied for 30 seconds decreased to 29.54 (SD 5.04) after the application, compared to 30.45 (SD 5.05) before. A corresponding t-test showed a significant difference $(p=0.019)$. Skin blood flow increased from a median of 0.76 (variance 0.062 ) before to a median of 0.86 (variance 0.16 ) after the procedure. The Wilcoxon rank test showed a significant difference ( $\mathrm{p}$ $=0.000$ ). Circulatory response was confirmed by SBP, DBP, and HR. SBP of $108.6 \mathrm{mmHg}$ (SD 14.8) before the study decreased to $105.7 \mathrm{mmHg}$ (SD 15.0) after the study, and DBP of $65.6 \mathrm{mmHg}$ (SD 11.1) before the study decreased to $62.7 \mathrm{mmHg}$ (SD 11.8) after the study. HR decreased from 71.6 beats per minute (SD 10.3) before to 69.2 beats per minute (SD 11.7) after. There was a significant difference in all cardiovascular indices $(\mathrm{p}<0.05)$. VAS (pain, stiffness, and fatigue) was significantly decreased after MVT $(\mathrm{p}<0.05)$. Conclusion: Micro Vibrational therapy tended to decrease muscle hardness and increase skin blood flow even in the short time of 30 seconds. The results suggest that local vibration stimulation is not likely to cause a sudden in-
\end{abstract}


crease in blood pressure or pulse rate fluctuation. These results suggest that hand vibration nursing care may be applicable to acute patients with unstable circulatory conditions.

\section{Keywords}

Micro Vibration Therapy, Nursing Care, Muscle Hardness, Skin Blood Flow, Immobility Patients

\section{Background}

Critically ill patients in intensive care units (ICU) are immobile due to bed rest during treatment [1] [2] [3]. Bed rest is a necessary treatment for critically ill patients because it promotes the recovery of organ functions and the reduction of metabolic resources or the reduction of pain and comfort of patients [4] [5] [6]. However, restful lying causes functional disability such as disuse muscle atrophy and joint contracture from an early stage [4] [7]. The mechanisms that cause muscle weakness in critically ill patients are complex and involve several interrelated processes [7] [8]. Skeletal muscle mass decreases by $1 \%-1.5 \%$ per day, and within a week, $10 \%-15 \%$ of muscle strength is lost [9] [10]. In addition, within two weeks, muscular contractures occur along with muscle atrophy [11] [12] [13]. By the immobility of intensive care unit, more than $1 / 3$ of patients who stay in the ICU for more than 2 weeks suffer from functional disabilities such as contractures of joints that are important for daily life, such as the knee, hip, and shoulder joints [14]. Furthermore, $69 \%$ of patients discharged from the ICU are associated with reduced activity of daily living (ADL) levels and delayed recovery [15] [16]. Recent studies have indicated that the therapeutic benefits of bed rest are outweighed by the long-term harms of delayed recovery and survival [1] [6] [9] [17] [18]. Early mobilization and exercise are recommended in the ICU [9]. Early mobilization of critically ill patients can stress and stimulate muscles, which can stimulate muscle protein synthesis pathways and inhibit catabolism [19]. Early weaning, in which patients move their bodies in parallel with treatment, can prevent muscle atrophy and joint contractures and prevent disuse syndrome [9]. Early mobilization of critically ill patients puts stress on muscles, and stimulation of muscles can stimulate muscle protein synthesis pathways and inhibit catabolism [19]. Early weaning, in which patients move their bodies in parallel with treatment, prevents muscle atrophy and joint contracture, and can prevent disuse syndrome.

In Japan, when the medical fees were revised in FY2018, "additional early bed release rehabilitation by multidisciplinary staff in ICU" [20] was newly established. However, in ICUs, there are multiple factors that inhibit early release, such as patient characteristics, lack of staff knowledge, and organizational culture [1]. In addition, it has been pointed out that the contents and systems of 
early rehabilitation vary greatly from facility to facility [21]. Early rehabilitation is started after the vital signs have been stabilized [22]. Therefore, it has been reported that it takes an average of 8.1 days to intervene [23]. During the period when early rehabilitation is not performed, muscle atrophy begins to occur, so it is important to move muscles altruistically to prevent it. Considering the characteristics of ICU patients, we focused on "Micro Vibration Therapy (MVT)," in which the nurse's hands are used to vibrate the muscles, from the perspective of promoting muscle extensibility safely and in a short time. With the hands of a nurse micro vibration has been used to improve joint contractures [24] [25].

However, the literature on Micro Vibration Therapy (MVT) has not scientifically proven the effects of Micro Vibration therapy on the body. In addition, since antigravity muscles are important in the process of Early mobilization, we will focus on the back, which is related to the erector spinae muscle, one of the antigravity muscles. The erector spinae muscles must maintain isometric/centrifugal contraction in order to support the head, which weighs $1 / 7$ of body weight.

\section{Purpose}

The purpose of this study is to develop nursing care that can prevent muscle atrophy in immobile patients in ICU using Micro Vibration Therapy (MVT). In this study, we will identify biological changes using muscle stiffness, skin blood flow, and circulatory response as indicators, and examine the possibility of adapting the therapy to acute patients. This study will contribute to the development of rehabilitation nursing in the acute phase.

\section{What Is Micro Vibration Therapy?}

Micro Vibration therapy was developed by Kamiya. The technique is positioned as a massage. In this technique, the nurse's hands are used to apply a certain amount of pressure to the target area, and the palms of the hands are vibrated from side to side in a fine manner, as if moving the muscles, rather than just rubbing the skin. In Japan, it is described as being effective in improving constipation and joint contractures in patients with disuse syndrome. It is often used for patients with disuse syndrome. It is not used outside Japan. Similar massages are vibration (shaking) and shaking (vibrating), but the difference is that the amplitude is greater and shaking applies stronger pressure than vibration [26]. The frequency and pressure of the two have not been scientifically proven, but it has been shown that the Micro Vibration Therapy is about $9 \mathrm{~Hz}$ and the pressure is about $94 \mathrm{mmHg}$ [27].

\section{Methods}

\subsection{Study Design}

This study is a before-and-after design to examine how the body changes after 30 seconds of Micro Vibration therapy which is one of the recovery programs for patients with disuse syndrome and is given by the nurse's hands. 


\subsection{Subject}

The subjects of this study were 30 healthy adults in their $20 \mathrm{~s}$ to $60 \mathrm{~s}$ with no problems in daily life. Subjects with diabetes mellitus, cardiovascular diseases, and back diseases were excluded. Participants were recruited using posters and the opportunity method.

\subsection{Environment Setting}

The environment was adjusted in order to eliminate the possibility that the environment might affect the measurement items. The room temperature was adjusted to $20^{\circ} \mathrm{C}-25^{\circ} \mathrm{C}$ by using an air-conditioning system one hour before the measurement. The temperature was checked with a multifunctional digital thermo-hygrometer (Therm Pro). As a result, the room temperature was $23^{\circ} \mathrm{C}$ $24^{\circ} \mathrm{C}$ and the humidity was $55 \%-59 \%$. The sound level was checked with a SOUND LEVEL METER (SM-325) to be less than $60 \mathrm{~dB}$ and was 50 - 56.8 Decibel. Illuminance was checked using a LIGHT METER (LM-332) and was 341 Lux. The practitioner stood on the right side of the bed and performed micro vibration with the right hand. The height of the bed was set at $45 \%$ of the height of the practitioner. The height of the bed was set at about $45 \%$ of the height of the practitioner. The reason for this is that this is the most efficient height for the work and does not put any burden on the back of the practitioner. The implementation was recorded using a digital video camera recorder (Canon iVIS HF R31). The camera was fixed on a tripod in a position where the subject's hand could be clearly seen under normal light.

\subsection{Preparing the Practitioner for Micro Vibrational Therapy}

Nurses who routinely administered Micro Vibrational therapy to patients were selected for this study.

These nurses were mentored by the developers. And the vibration frequency and hand pressure of these nurses' techniques are consistent [27].

Before implementation, we checked the practitioner's standing position, hand application method, bed height, and implementation time (30 seconds).

\subsection{Subject Preparation Conditions}

1) In order to eliminate the influence on the measurement items, we explained that the participants should refrain from drinking alcohol 8 hours prior to the start of the measurement, consuming caffeine-rich or stimulating beverages on the day of the measurement, eating a meal 1 hour prior to the start of the measurement, and engaging in strenuous exercise.

2) Age, gender, height, weight, Barthel Index (basic activities of daily living), pain, stiffness, and fatigue were surveyed using a visual analog scale (VAS).

3) The subjects were dressed and prepared in a non-constricting nightgown (Nagai Leven SG-301) with an open back.

4) A marker (NITOMS Yu-Ki-Ban non-woven fabric) was placed on the skin 
$2 \mathrm{~cm}$ above the area where muscle hardness and blood flow were to be measured.

\subsection{Survey Items and Procedures}

\subsubsection{Survey Items}

1) Attributes: Age, gender, height, weight, and Barthel Index (basic activities of daily living) were surveyed.

2) Muscle hardness: The NEUTONE TDM-N1 muscle hardness tester was used. The accuracy of this device was checked by measuring it three times because it is manually operated.

3) Skin blood flow: For skin blood flow, we used a laser (tissue) blood flow meter Omega Flow contact type (FLO-C1). This is a general laser blood flowmeter that measures blood flow by placing an optical fiber probe in contact with a living body. Since the probe is small (10 $\mathrm{mm}$ in diameter), a tape with holes was attached to the measurement site to prevent shifting of the measurement site.

4) Circulatory dynamics: Blood pressure and pulse were used as indicators of circulatory dynamics. Systolic blood pressure (SBP), diastolic blood pressure (DBP), and pulse rate (HR) were measured on the left upper arm of all subjects. The OMRON HCR-7106 brachial blood pressure monitor was used for measurement.

5) Subjective sensation: The subject's subjective sensation of pain, stiffness, and fatigue were investigated using the VAS scale $(0.0-10.0 \mathrm{~cm})$ before and after the 1 Micro Vibration Therapy.

\subsubsection{Procedure Protocols (Figure 1)}

1) The subject was placed on a bed in the measurement room in a stable posture in the right lateral supine position and rested for 5 minutes, assuming that the test would be performed during repositioning of immobile patients for whom early rehabilitation is difficult in the acute phase.

2) Muscle hardness, skin blood flow, blood pressure, and pulse rate were measured in this order.

3) Micro Vibration Therapy was performed for 30 seconds, referring to the duration of Micro Vibration Therapy performed on actual patients.

4) In this study, we chose the vastus lateralis muscle of the back as the application site for MicroVibration Therapy. In fact, micro-vibration therapy is performed on the back of patients with chronic conditions. This is because it is a part of the body that moves relatively little. Also, it is easy to measure the changes of MicroVibration Therapy.

5) Skin blood flow, Muscle hardness, Blood pressure, and Heart rate were measured in this order after Micro Vibration therapy.

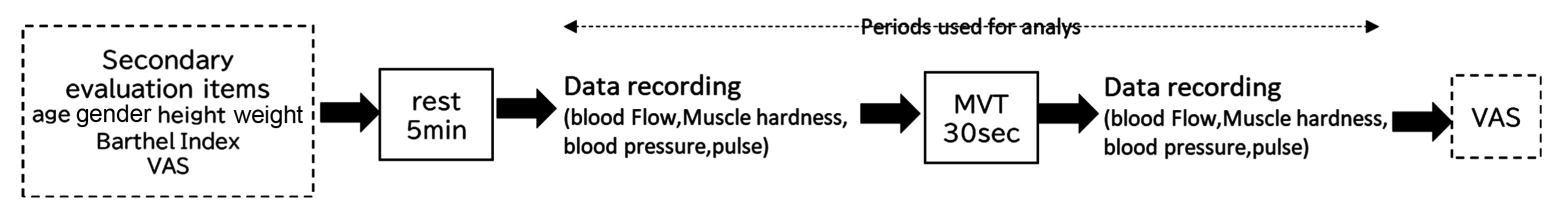

Figure 1. The experiment protocol for sampling data. 


\subsection{Analysis Method Data Analysis}

IBM SPSS Statistics 27 was used for all statistical analyses, and the significance level was set at $5 \%$. Descriptive statistics were calculated for all data. Age, height, weight, BMI, muscle hardness, and skin blood flow were checked for normality using the Shapiro-wilk test. Corresponding t-test and Wilcoxon rank test were conducted with the control group before and after the Micro Vibration therapy. The Mann-Whitney $U$ test was used to determine the relationship with attributes.

\subsection{Ethical Considerations}

This study was conducted under the approval of the Ethics Committee of Osaka Medical and Pharmaceutical University. (Approval number: 2020-119).

This study was conducted in accordance with the ethical principles for research involving human subjects set forth in the Declaration of Helsinki.

The subjects were informed in writing and orally about the contents of the study, that they were free to participate or withdraw from the study, and that they could withdraw their intention to participate even after cooperating in the study. The consent form was signed by the subjects to confirm their intention.

\section{Result}

\subsection{Baseline Characteristics of Participants (Table 1)}

There were 30 subjects who participated in the study. The gender of the subjects was 22 females and 8 males. Age was 29.3 years (median 26.0 years, range 22 - 56 years), height was $160.7 \pm 10.1 \mathrm{~cm}$ (median $158.0 \mathrm{~cm}$, range $138.0-183.0 \mathrm{~cm}$ ), weight was $56.1 \pm 9.4 \mathrm{~kg}$ (median 56.0 range $38.0-87.0 \mathrm{~kg}$ ). In order to check whether the patients had any difficulty in daily life, we checked their activities of daily living with the Barthel Index. All were scored 100/100. The results of Shapiro-wilk test for each attribute and item showed no normality for age, VAS, and skin blood flow after MVT, so the analysis was based on non-parametric for that item.

\subsection{Muscle Hardness}

Muscle hardness was measured three times each before and after the test to increase reliability. Intraclass correlation coefficient (ICC) was calculated for intra-examiner reliability $(1,1)$ and average reliability $(1,3)$ of the three measurements. In addition, the coefficient of variation (CV) was used to check the variability of the data. As a result, the ICCs before implementation $(1,1)$ were 0.95 and ICCs after implementation $(1,3)$ were 0.98 (ICCs are results exceeding 0.9, indicating "excellent reliability"). The mean coefficient of variation was 0.04 (range 0.00 - 0.08). The post-implementation ICC $(1,1)$ was 0.95 and ICC $(1,3)$ was 0.98 (Table 2). The mean coefficient of variation was 0.03 (range 0.00 0.07). The measurement error was very low. Comparing the pre- and post-means of the 30 participants, the pre-measurement was 30.45 (SD 5.05) while the post-measurement was 29.54 (SD 5.04). The corresponding t-test showed a sig- 
nificant difference $(\mathrm{p}=0.019)$ (Figure 2).

Nine patients had an increase of 0.3 to 4.7 score and 20 patients had a decrease of 0.3 to 5.3 score. 1 patient had no change. The Mann-Whitney-U test was used to analyze each attribute, with the unchanged group as the ascending group and the decreasing group as the dependent variable. As a result, there was no significant difference in comparison with attributes such as age, gender, height, weight, and BMI (Table 3).

Table 1. Baseline characteristics of participants. Values are mean \pm standard deviation, median and range.

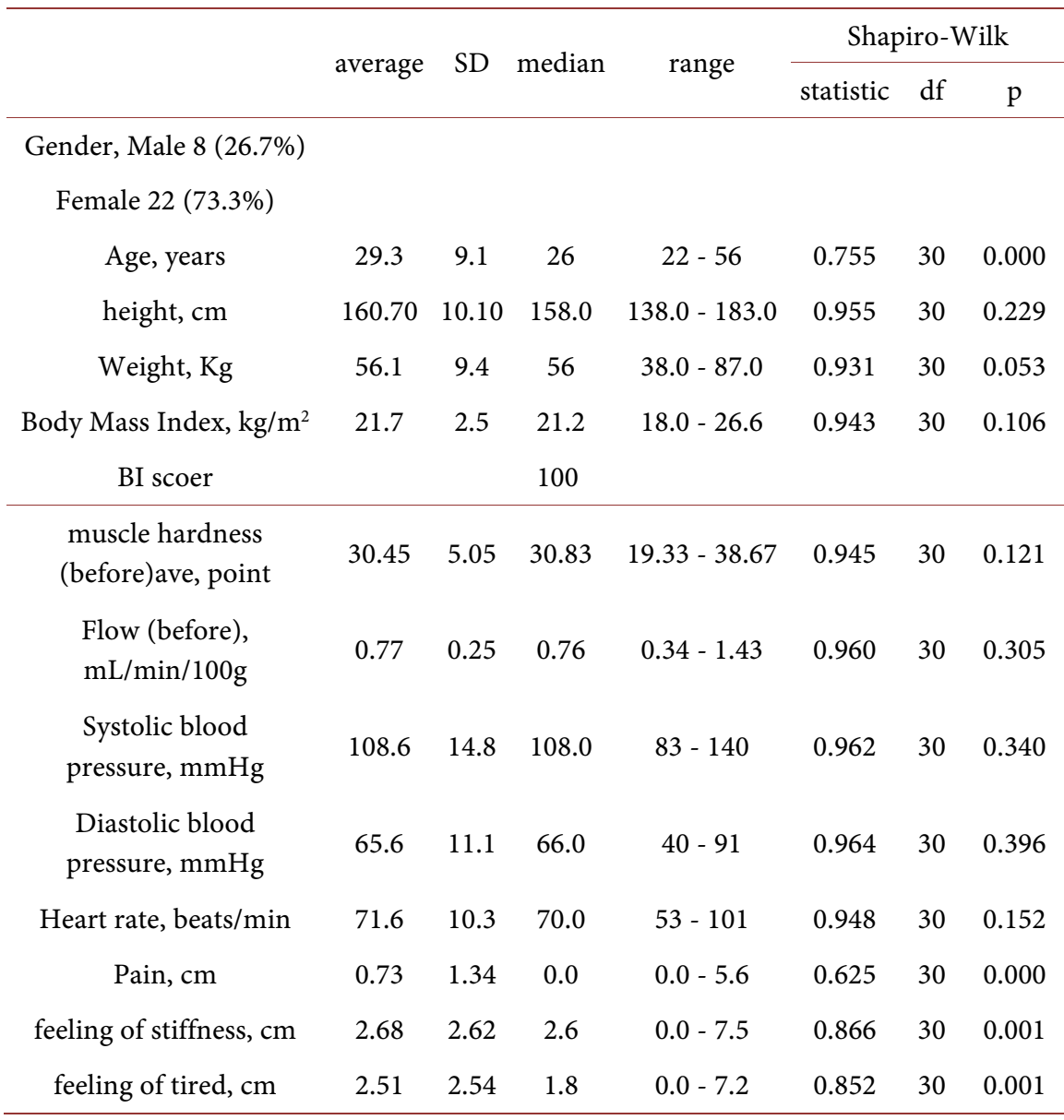

Table 2. Intraclass correlation coefficient in 3 measurements of muscle hardness. ICC estimates and their 95\% confident intervals were calculated using SPSS statistical package version based on a mean-rating $(\mathrm{k}=3)$, absolute-agreement, 2-way mixed-effects model.

\begin{tabular}{|c|c|c|c|c|c|c|c|c|c|}
\hline & & \multirow{2}{*}{$\begin{array}{l}\text { Intraclass } \\
\text { correlation }\end{array}$} & \multicolumn{2}{|c|}{ 95\% Confidence interval } & \multicolumn{4}{|c|}{ F Test With True Value 0} & \multirow{2}{*}{$\begin{array}{l}\text { coefficient } \\
\text { of variation }\end{array}$} \\
\hline & & & Lower Bound & Upper Bound & Value & df 1 & df 2 & $\mathrm{p}$ & \\
\hline \multirow{2}{*}{ preMVT } & $\operatorname{ICC}(1,1)$ & 0.947 & 0.906 & 0.972 & 54.631 & 29 & 60 & 0.000 & \multirow{2}{*}{0.03} \\
\hline & $\operatorname{ICC}(1,3)$ & 0.982 & 0.967 & 0.991 & 54.631 & 29 & 60 & 0.000 & \\
\hline \multirow{2}{*}{ postMVT } & $\operatorname{ICC}(1,1)$ & 0.949 & 0.909 & 0.973 & 56.383 & 29 & 60 & 0.000 & \multirow{2}{*}{0.04} \\
\hline & $\operatorname{ICC}(1,3)$ & 0.982 & 0.968 & 0.991 & 56.383 & 29 & 60 & 0.000 & \\
\hline
\end{tabular}




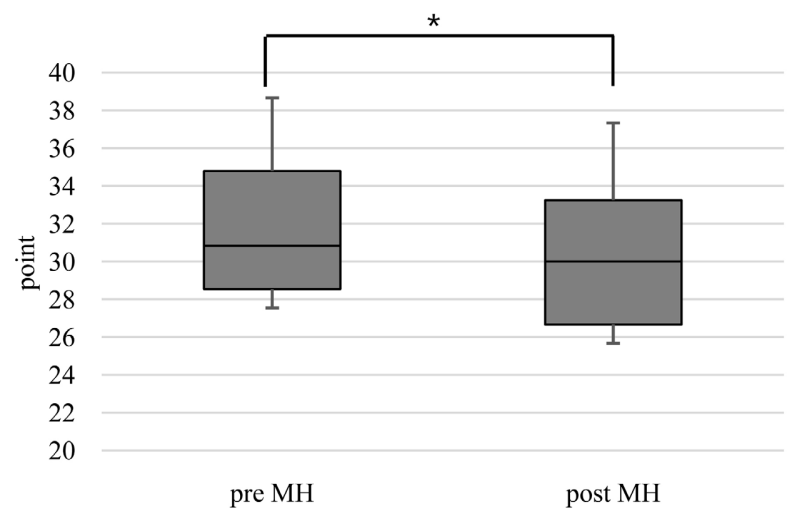

Figure 2. Boxplot of MH (muscle hardness) previous and post MVT (box: median and interquartile range; whiskers: minimum and maximum).

Table 3. Each attribute (age, gender, height, weight, and BMI) was analyzed by MannWhitney-U test, with the addition of one person with no change as the dependent variable for the increased muscle hardness group and the decreased group as the dependent variable.

\begin{tabular}{|c|c|c|c|c|c|c|}
\hline & & $\mathrm{n}$ & $\begin{array}{c}\text { average } \\
\text { rank }\end{array}$ & $\begin{array}{l}\text { rank } \\
\text { sum }\end{array}$ & Mann-Whitney U & $\mathrm{p}$ \\
\hline \multirow[t]{2}{*}{ tall } & $\begin{array}{l}\text { Group to } \\
\text { decrease }\end{array}$ & 20 & 15.83 & 316.50 & 93.500 & 0.774 \\
\hline & Increased group & 10 & 14.85 & 148.50 & & \\
\hline \multirow[t]{2}{*}{ weight } & $\begin{array}{l}\text { Group to } \\
\text { decrease }\end{array}$ & 20 & 17.25 & 345.00 & 65.000 & 0.123 \\
\hline & Increased group & 10 & 12.00 & 120.00 & & \\
\hline \multirow[t]{2}{*}{ gender } & $\begin{array}{l}\text { Group to } \\
\text { decrease }\end{array}$ & 20 & 15.75 & 315.00 & 95.000 & 0.774 \\
\hline & Increased group & 10 & 15.00 & 150.00 & & \\
\hline \multirow{2}{*}{ age } & $\begin{array}{l}\text { Group to } \\
\text { decrease }\end{array}$ & 20 & 15.25 & 305.00 & 95.000 & 0.823 \\
\hline & Increased group & 10 & 16.00 & 160.00 & & \\
\hline \multirow[t]{2}{*}{ BMI } & $\begin{array}{l}\text { Group to } \\
\text { decrease }\end{array}$ & 20 & 17.28 & 345.50 & 64.500 & 0.118 \\
\hline & Increased group & 10 & 11.95 & 119.50 & & \\
\hline
\end{tabular}

\subsection{Skin Blood Flow}

Skin blood flow was measured before and after the Micro Vibration therapy. The sensor used in this study (Laser Tissue Blood Flowmeter) is capable of measuring three factors (FLOW, MASS, and VELOCITY). The principle of measurement is that the laser beam of the blood flowmeter is irradiated into the tissue, and the incident light spreads out while repeatedly scattering and reflecting. Among them, the light hitting the flowing blood undergoes frequency fluctuation (Doppler shift) and changes its frequency according to the speed of the 
blood flow. On the other hand, light scattered only by stationary blood does not undergo frequency fluctuation and its frequency remains unchanged. Some of these lights are captured by the light receiving fiber of the blood flow meter. The frequency modulation of the frequency-modulated light corresponds to the blood flow velocity component (VELOCITY), and the intensity of the captured light corresponds to the red blood cell mass component (MASS). The product of these two parameters (VELOCITY and MASS) corresponds to the tissue blood flow rate (FLOW). Therefore, FLOW will be the target of analysis in this experiment. Since the blood flow rate before and after vibration was to be measured, a contact type was used and applied directly to the skin of the measurement site. 10 seconds of data was sampled and collected. As a result, the median value was 0.76 (variance 0.062 ) before implementation and 0.86 (variance 0.16 ) after implementation. 24 out of 30 subjects had an increase of $98.85 \%-2.6 \%$ and 6 had a decrease of $0.97 \%-13.11 \%$. Wilcoxson signed rank test showed a significant increase $(\mathrm{p}=0.000)$. (Figure 3$)$

\subsection{Circulatory Response}

Blood pressure and pulse rate were measured to capture the changes in circulatory dynamics. The mean values of SBP, DBP, and HR before and after Micro Vibration therapy were calculated. SBP before implementation was $108.6 \mathrm{mmHg}$ (SD 14.8), while after implementation it was $105.7 \mathrm{mmHg}(\mathrm{SD} \mathrm{15.0)}(\mathrm{p}=0.034)$. DBP before the study was $65.6 \mathrm{mmHg}$ (SD 11.1), while after the study it was 62.7 mmHg (SD 11.8); HR before the study was 71.6 beats per minute (SD 10.3), while after the study it was 69.2 beats per minute (SD 11.7). $(p=0.005)$. (Figure 4, Table 4)

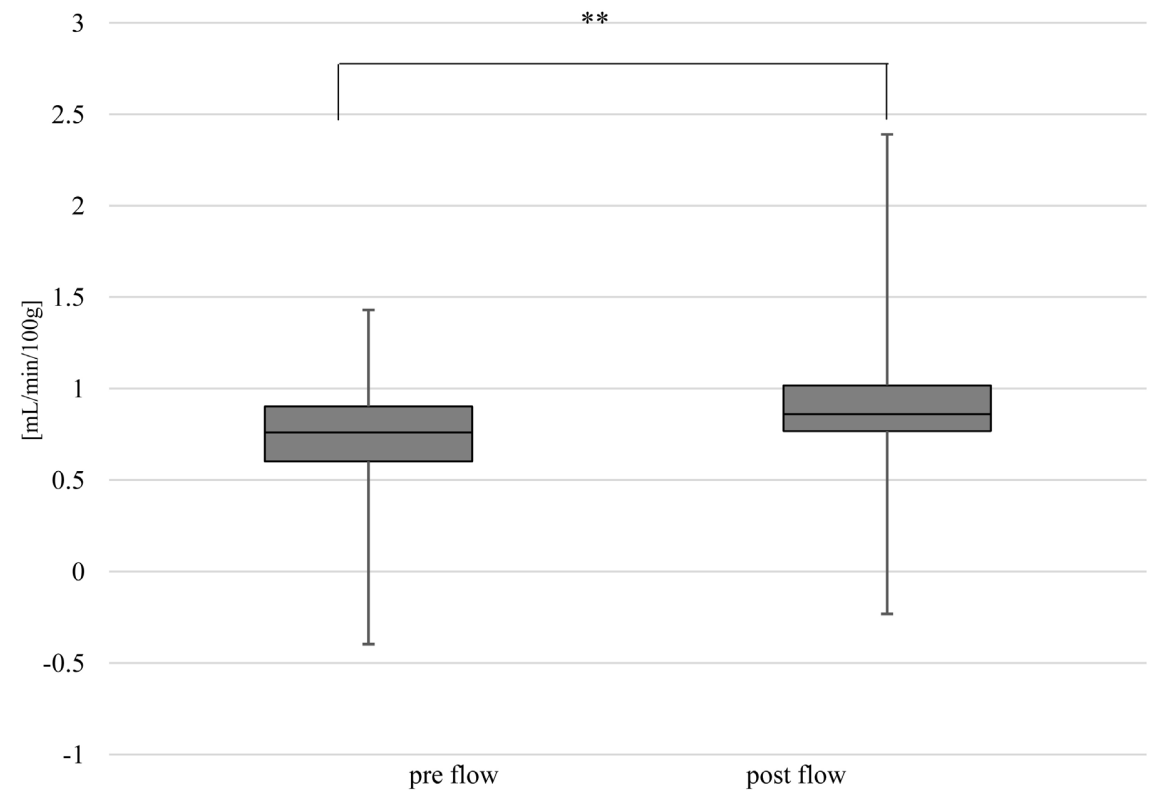

Figure 3. Boxplots of (skin blood flow) before and after MVT (boxes: median and interquartile range; whiskers: minimum and maximum values). 
Table 4. Comparison of mean values of SBP, DBP, and HR previos and post MVT. SBP, DBP, and HR were analyzed by corresponding t-test.

\begin{tabular}{cccccccccc}
\hline & \multicolumn{9}{c}{$95 \%$ Confidence interval } \\
\cline { 2 - 9 } & Average & $\begin{array}{c}\text { Standard } \\
\text { deviation }\end{array}$ & $\begin{array}{c}\text { Standard error } \\
\text { of the mean value }\end{array}$ & $\begin{array}{c}\text { Lower } \\
\text { Bound }\end{array}$ & $\begin{array}{c}\text { Upper } \\
\text { Bound }\end{array}$ & $\mathrm{t}$ & $\mathrm{Df}$ & $\mathrm{p}$ \\
\hline pre SBP - post SBP & 2.83 & 6.98 & 1.27 & 0.23 & 5.44 & 2.22 & 29.00 & 0.03 \\
pre DBP - post DBP & 2.90 & 4.99 & 0.91 & 1.04 & 4.76 & 3.18 & 29.00 & 0.00 \\
pre HR - post HR & 2.37 & 4.28 & 0.78 & 0.77 & 3.96 & 3.03 & 29.00 & 0.01 \\
\hline
\end{tabular}

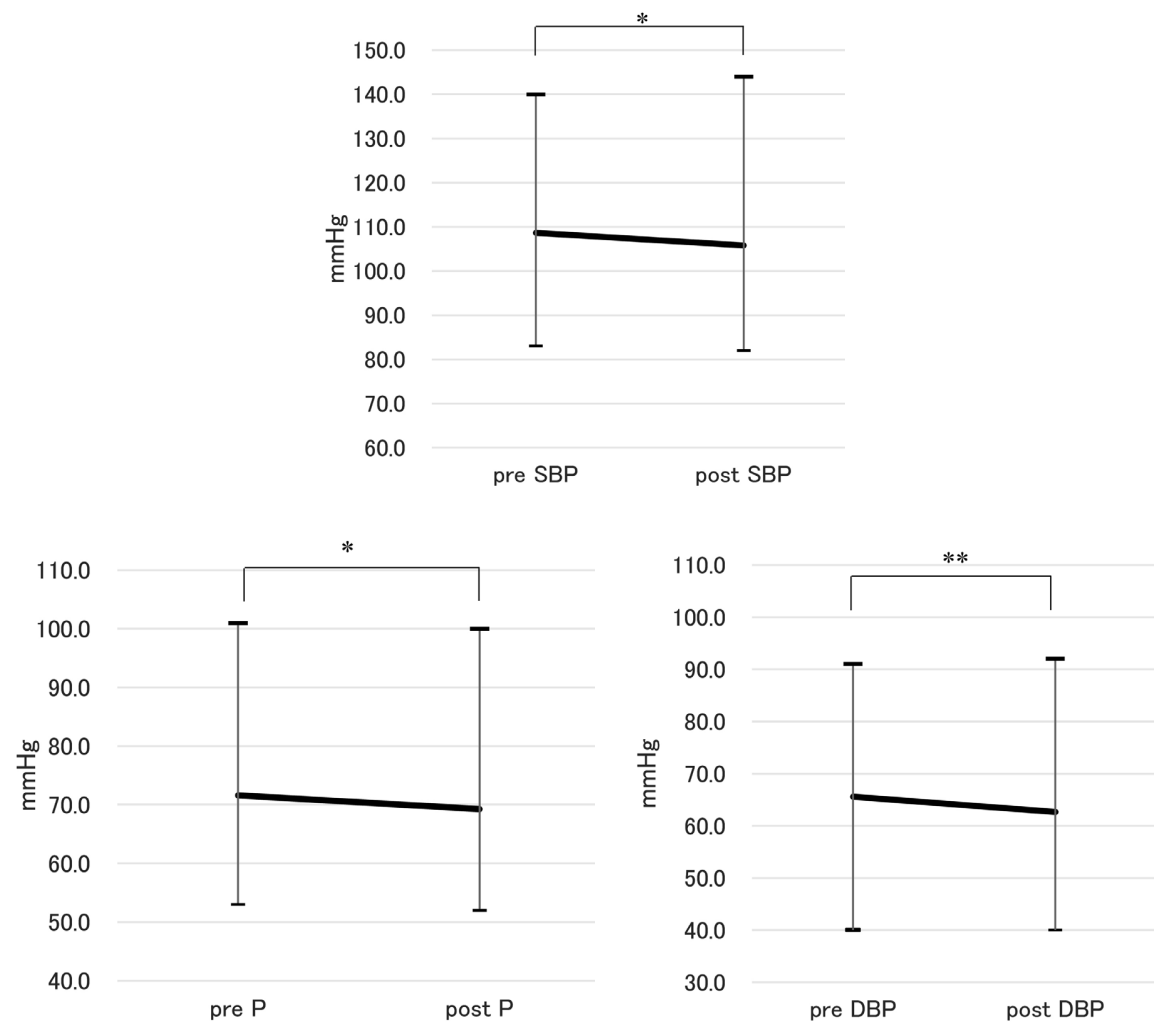

Figure 4. Comparison of SBP, DBP, and HR averages. Error bars indicate the maximum and minimum values. All items were significantly decreased after MVT.

\subsection{Subjective Impressions}

In the VAS, participants were asked to mark their current sensations of pain, stiffness, and fatigue on a horizontal straight line of $100 \mathrm{~mm}$ in length. The left end of the line was defined as "none at all" and the right end as "severe/worst sensation," and the distance from the left to the mark was measured. Since the data of all three items were not normally distributed, the difference was confirmed by Wilcoxon rank test. As a result, pain was $0.73 \mathrm{~cm}$ before implementation and $0.50 \mathrm{~cm}$ after implementation $(\mathrm{p}=0.026)$. Stiffness was $2.68 \mathrm{~cm}$ before and $2.14 \mathrm{~cm}$ after the test $(\mathrm{p}=0.006)$. Fatigue was $1.76 \mathrm{~cm}$ after the test compared to $2.51 \mathrm{~cm}$ before the test $(\mathrm{p}=0.000)$. A significant decrease was observed in all items (Figure 5). 


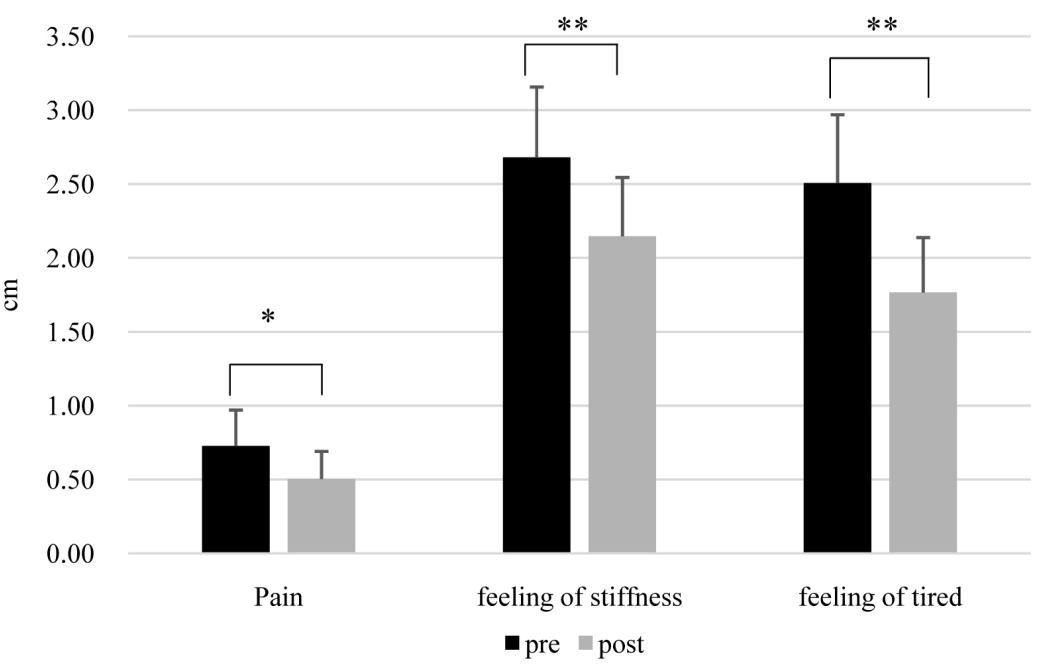

Figure 5. Subjective indices were confirmed by VAS (Visual Analog Scale). The subjects were asked to mark the sensations of "pain," "stiffness," and "fatigue" on a horizontal line of $100 \mathrm{~mm}$ in length. Black bars indicate the baseline. Gray bars indicate after MVT. Differences were confirmed by Wilcoxon rank test. A significant decrease was observed for all items $(\mathrm{p}<0.05)$.

\section{Discussion}

\subsection{Changes in Muscle Hardness}

Muscle hardness is perceived as the elastic stiffness of a muscle [28] [29]. If the hardness is high, the muscle generally feels hard to the touch; if it is low, the muscle feels soft. Muscle hardness is significantly correlated with extensibility. If a muscle is stiff and difficult to stretch, not only will it not be able to perform well, but it will also be painful and restrict movement. Such a condition leads to immobility. Immobility leads to decreased blood flow and nutrients at the tissue level, and at the cellular level, it causes metabolic changes in substrate synthesis [11]. When muscle is under moderate tension, fibroblasts synthesize more collagen and produce more tissue stretch [30]. When a critically ill patient recovers and is ready to move, if the muscle is stiff and difficult to stretch, it may be difficult to release. Stretching and heat therapy to improve extensibility may be too much for critically ill patients. The advantage of Micro Vibration therapy is that it is simple and can be done anytime and anywhere, since it uses the nurse's hands. Even when a critically ill patient is immobile before leaving the bed, the nurse's hands can be used to apply vibration easily before the muscles become stiff, which may help maintain muscle tone in the back. In this study, 30 seconds of Micro Vibration therapy was used, but if it was longer than 30 seconds, the number of vibrations would be too small or the hands would get tired and apply too much pressure. Although only 30 seconds is considered to be a small amount, the muscle hardness decreased significantly from 30.45 (SD 5.05) to 29.54 (SD 5.04) after implementation. This suggests that a small number of frequent stimulation may prevent overuse. Considering that we aim to adapt the method to critically ill patients in the future, overuse of the muscle, such as the so-called 
rubbing, is detrimental. To prevent overuse, training with low load and high frequency is effective [31]. Micro Vibration Therapy is low load as it is performed for 30 seconds and may effectively promote muscle stretching by performing it many times a day.

The body mass of the subjects in this study ranged from 18.0 to $26.6 \mathrm{~kg} / \mathrm{m}^{2}$ BMI. BMI and muscle hardness were compared to determine the influence of other tissues such as subcutaneous fat. The results of a study using a similar instrument also reported that muscle hardness could be measured without considering the amount of fat, as high BMI does not necessarily result in low muscle hardness due to fat thickness [32] [33]. They also stated that the reason why muscle hardness differs among subjects even under the same conditions is individual differences [33]. We believe that the results of the present study for a broad BMI population support previous studies.

Vibration, if too strong, can cause adverse events, but mild vibration is considered beneficial and is used in medicine [34]. Previous studies applying strong vibrations of 50 or $100 \mathrm{~Hz}$ to living organisms have shown that whole-body vibrations stimulate muscle spindles and alpha-motor neurons, causing muscle contraction, increasing muscle strength, improving balance, and increasing bone mass [35]. Even when localized vibration is applied, it improves muscle strength [36]. As a result, it has shown the potential to enhance muscle strength and sports performance [35] [36] [37]. In addition, studies investigating the effects of vibration on flexibility and stiffness [38] [39] found that most of the vibrations were between 25 and $50 \mathrm{~Hz}$. The stimuli improved flexibility. On the other hand, the studies that did not improve flexibility indicated that the subjects were older and that high frequency vibration of $40 \mathrm{~Hz}$ may have affected the muscles. [38].

The hand vibration is a weak vibration of about $9 \mathrm{~Hz}$. It is characterized by the addition of pressure of about $94 \mathrm{mmHg}$ along with the vibration. [27]. It is possible that the muscle hardness decreased due to the stretching effect caused by the application of pressure along with the vibration.

Micro Vibration therapy is not intended to improve muscle strength in the first place, but to relax stiff muscles, increase stretch, and possibly prevent muscle atrophy. If vibration can improve muscle strength, there is room to consider whether hand vibration can also increase muscle strength.

\subsection{Change in Skin Blood Flow}

Several mechanisms by which vibration stimulation causes changes in the body have been clarified. One of the effects of vibration on the body is to increase blood circulation and generate more heat, which promotes flexibility [38]. Vibrations also cause muscles to contract and relax, raising the pain threshold during stretching [38]. Otherwise, when vibration is transmitted to the skin, mechanical stress is generated, which increases skin blood flow by promoting NO (nitric oxide) production from vascular endothelial cells [40]. When blood flow increases and shear stress acting on vascular endothelial cells increases, vasodilator substances nitric oxide and prostacyclin are produced and vasodilation 
occurs [41]. In contrast to the studies by Nakagami and Ichioka, which used mechanical vibrations, the present study used the hands of a nurse, which resulted in weak vibrations of approximately $9 \mathrm{~Hz}$. This is about one-fifth that of a typical home massage device. Although the vibration frequency was much weaker than that of the previous study, we confirmed an increase in skin blood flow. Restful lying, which is used for critically ill patients, often results in a state of skeletal muscle immobility, and such a state causes vascular endothelial cells to be injured due to increased oxidative stress from the generation of oxidative enzyme active species in skeletal muscle, resulting in degenerative changes in capillaries [42] [43]. Vibrations increase NO release from vascular endothelial cells, causing adjacent vascular smooth muscle cells to dilate the vessels. When blood vessels are dilated, blood flow to soft tissues is increased. Previous studies have shown results with stronger vibrations than Micro Vibration therapy, but given that the increase was also seen with weaker vibrations, this may be an effective care for immobile patients. Especially in critically ill patients who are immobile, the gravity of the patient's own body tends to be applied to the back of the body, causing insufficient blood flow. Increasing skin blood flow by vibration has the potential to prevent this from happening.

\subsection{Changes in Circulatory Response}

Vibration and shaking used in medical massage are contraindicated in patients with hypertension or coronary artery disease because of the risk of increased blood pressure [26]. In this study, it was assumed that the vibration stimulation was local and would not cause any change in circulatory dynamics. However, we observed a decrease in both systolic and diastolic blood pressure and pulse rate. Systolic blood pressure decreased by $2.7 \%$, diastolic blood pressure by $4.4 \%$, and pulse rate by $3.4 \%$. Because the study design was based on healthy adults, it is possible that the gravitational resistance decreased over time, causing the smooth muscle in the small arteries to relax. Alternatively, the hand vibration may have been a pleasant stimulus, and the parasympathetic nervous system may have been activated. Considering the future application of this Micro Vibration therapy to critically ill patients, blood pressure and pulse rate fluctuations are important factors. After 3 days of resting and lying in bed, circulating plasma volume decreases by $8 \%-10 \%$ [44]; after 3 weeks, the cardiac pressure reflex to central stimulation of the carotid baroreceptors decreases by $30 \%$ [45]. The circulatory dynamics, which also have a significant effect on early weaning, can be altered by turning if the patient is in a prolonged resting bed state [44]. In a study of people without cardiac disease, the effect of changes in body position on the size and shape of the inferior vena cava was examined. They reported that the inferior vena cava was smallest in the left lateral supine position. The present study was conducted in the right lateral supine position. The left lateral supine position was not examined, but considering the possibility of further reduction, the right lateral supine position may be more suitable than the left lateral supine position for adaptation to critically ill patients. 


\subsection{Changes in Subjective Sensation}

The subjective sensation was evaluated by VAS in order to confirm the sensory effects of Micro Vibration therapy on the subject. As a result, a significant decrease was observed in all items. We thought that the vibration to the back might cause some discomfort, but none of the subjects showed any discomfort. Although we could not deny the possibility that the vibration might spill over to the head, the fact that there was no increase in pain or fatigue suggests that no force was applied to fix the head. However, the fact that there was no increase in pain or fatigue suggests that no force was applied to immobilize the head. The fact that there was a decrease in stiffness in the area where Micro Vibration therapy was applied suggests that no adverse events occurred. There has been no research on the subjective sensation brought about by Micro Vibration therapy. The fact that pain and stiffness were reduced in this study suggests that Micro Vibration Therapy may provide pleasant stimulation. Studies investigating the correlation between subjective shoulder stiffness and muscle hardness have reported that the degree of subjective pain and stiffness does not necessarily coincide with muscle hardness [46] [47] [48] [49]. We believe that the reason why the present study differed from those studies is that the intervention of 30 seconds of Micro Vibration Therapy may have resulted in a decrease in muscle hardness and subjective sensation, whereas the previous studies compared subjective sensation at a single point and muscle hardness. On the other hand, since the heart rate decreased with the passage of the study time and the parasympathetic nervous system became dominant, the subjects may not have simply felt unwell. Therefore, in the future, it is necessary to add not only VAS but also heart rate variability analysis, which is an indicator of the autonomic nervous system, to examine whether it can really be a pleasant stimulus.

\section{Conclusion}

In this study, we compared and examined each item based on the hypothesis that the muscle hardness of the back decreases and the skin blood flow increases in healthy adults when they perform Micro Vibration Therapy. The results showed that 30 seconds of Micro Vibration Therapy tended to decrease muscle hardness and increase skin blood flow. This suggests that Micro Vibration Therapy promotes muscle elasticity and blood flow, and may prevent disuse. In addition, the changes in circulatory dynamics before and after the application of Micro Vibration therapy were examined in terms of changes in blood pressure and pulse rate. As a result, it was suggested that the increase in local skin blood flow on the back did not affect the circulatory dynamics, but that the decrease in blood pressure and the position of the patient should be taken into consideration when administering this treatment to a severely ill person.

\section{Limitations of the Study}

The present study was conducted to compare before and after the implementa- 
tion of Micro Vibration Therapy. The subjects were in their $20 \mathrm{~s}$ to $50 \mathrm{~s}$, and the changes in muscle hardness and skin blood flow were confirmed for a wide range of ages and body sizes. One of the limitations of this study is that it was conducted on healthy adults who did not have muscle atrophy. Whether the changes induced by the Micro Vibration Therapy intervention are similar in severely ill patients should be examined. In addition, many of the patients who are currently receiving Micro Vibration therapy nursing care in Japan are elderly. Since measurements have not been conducted on elderly patients or patients with little muscle thickness or subcutaneous tissue, it is necessary to verify the results in the future. In addition, this study was limited to a pre/post comparison of one group; knowing how long the changes in muscle hardness and skin blood flow last after 30 seconds of Micro Vibration Therapy nursing care can be used as a guideline for the number of times per day it should be performed. In actual clinical situations, Micro Vibration Therapy is performed many times a day. In addition, the patient receives nursing care that stimulates the muscles, such as repositioning and wiping, and it is necessary to examine the results before and after the implementation of Micro Vibration Therapy nursing care when these are added.

\section{Future Prospects Future Scope}

In this study, we focused on whether Micro Vibration Therapy decreases muscle hardness and increases skin blood flow in the back, but in the future, it is necessary to investigate the motor performance of the lower limbs by applying it to other areas related to leaving the bed, such as the lower limbs (quadriceps femoris and gastrocnemius). In addition to muscle hardness and skin blood flow, changes in cerebral blood flow and autonomic nervous system should be confirmed, and other indices that may cause physiological responses should also be examined to see how these responses change in critically ill patients. Since we are currently administering Micro Vibration Therapy to patients with disuse syndrome, we would like to examine the effects of other nursing interventions.

\section{Acknowledgements}

The authors would like to thank all the subjects who participated in this study.

And also to the clinical nurses with professional MVT skills for providing the MVT.

I thank Professor Emeritus Katsuko Kamiya, the developer of MVT, for her kind advice. And we thank Professor Chiharu Akazawa for his kind advice in editing and improving the manuscript.

There are no conflicts of interest that should be disclosed. This study was supported by a grant from Tenri Healthcre University for Young Investigators.

\section{Conflicts of Interest}

The authors declare no conflicts of interest regarding the publication of this paper. 


\section{References}

[1] Dubb, R., Nydahl, P., Hermes, C., Schwabbauer, N., Toonstra, A., Parker, A.M., Kaltwasser, A. and Needham, D.M. (2016) Barriers and Strategies for Early Mobilization of Patients in Intensive Care Units. Annals of the American Thoracic Society, 13, 724-730. https://doi.org/10.1513/AnnalsATS.201509-586CME

[2] Chambers, M.A., Moylan, J.S. and Reid, M.B. (2009) Physical Inactivity and Muscle Weakness in the Critically Ill. Critical Care Medicine, 37, S337-S346. https://doi.org/10.1097/CCM.0b013e3181b6e974

[3] Dittmer, D.K. and Teasell, R. (1993) Complications of Immobilization and Bed Rest. Part 1: Musculoskeletal and Cardiovascular Complications. Canadian Family Physician, 39, 1428-1432, 1435-1437.

[4] Brower, R.G. (2009) Consequences of Bed Rest. Critical Care Medicine, 37, S422S428. https://doi.org/10.1097/CCM.0b013e3181b6e30a

[5] Fuest, K. and Schaller, S.J. (2018) Recent Evidence on Early Mobilization in Critical-Ill Patients. Current Opinion in Anaesthesiology, 31, 144-150. https://doi.org/10.1097/ACO.0000000000000568

[6] Lipshutz, A.K. and Gropper, M.A. (2013) Acquired Neuromuscular Weakness and Early Mobilization in the Intensive Care Unit. Anesthesiology, 118, 202-15. https://doi.org/10.1097/ALN.0b013e31826be693

[7] Truong, A.D., Fan, E., Brower, R.G. and Needham, D.M. (2009) Bench-to-Bedside Review: Mobilizing Patients in the Intensive Care Unit-From Pathophysiology to Clinical Trials. Critical Care, 13, Article No. 216. https://doi.org/10.1186/cc7885

[8] Gruther, W., Benesch, T., Zorn, C., Paternostro-Sluga, T., Quittan, M., FialkaMoser, V., Spiss, C., Kainberger, F. and Crevenna, R. (2008) Muscle Wasting in Intensive Care Patients: Ultrasound Observation of the $M$. quadriceps Femoris Muscle Layer. Journal of Rehabilitation Medicine, 40, 185-189. https://doi.org/10.2340/16501977-0139

[9] Hashem, M.D., Nelliot, A. and Needham, D.M. (2016) Early Mobilization and Rehabilitation in the ICU: Moving Back to the Future. Respiratory Care, 61, 971-979. https://doi.org/10.4187/respcare.04741

[10] Lach, H.W., Lorenz, R.A. and L'Ecuyer, K.M. (2014) Aging Muscles and Joints: Mobilization. Critical Care Nursing Clinics of North America, 26, 105-113. https://doi.org/10.1016/j.ccell.2013.10.005

[11] Akeson, W.H., Amiel, D., Abel, M.F., Garfin, S.R. and Woo, S.L. (1987) Effects of Immobilization on Joints. Clinical Orthopaedics and Related Research, 219, 28-37. https://doi.org/10.1097/00003086-198706000-00006

[12] Trudel, G. and Uhthoff, H.K. (2000) Contractures Secondary to Immobility: Is the Restriction Articular or Muscular? An Experimental Longitudinal Study in the Rat Knee. Archives of Physical Medicine and Rehabilitation, 81, 6-13. https://doi.org/10.1016/S0003-9993(00)90213-2

[13] Diekstall, P., Schulze, W. and Noack, W. (1995) Immobilization Damage. Sportverletzung Sportschaden, 9, 35-43. https://doi.org/10.1055/s-2007-993420

[14] Clavet, H., Hebert, P.C., Fergusson, D., Doucette, S. and Trudel, G. (2008) Joint Contracture Following Prolonged Stay in the Intensive Care Unit. Canadian Medical Association Journal, 178, 691-697. https://doi.org/10.1503/cmaj.071056

[15] Adler, J. and Malone, D. (2012) Early Mobilization in the Intensive Care Unit: A Systematic Review. Cardiopulmonary Physical Therapy Journal, 23, 5-13. https://doi.org/10.1097/01823246-201223010-00002 
[16] van der Schaaf, M., Beelen, A., Dongelmans, D.A., Vroom, M.B. and Nollet, F. (2009) Functional Status after Intensive Care: A Challenge for Rehabilitation Professionals to Improve Outcome. Journal of Rehabilitation Medicine, 41, 360-366. https://doi.org/10.2340/16501977-0333

[17] Buckwalter, J.A. (1996) Effects of Early Motion on Healing of Musculoskeletal Tissues. Hand Clinics, 12, 13-24. https://doi.org/10.1016/S0749-0712(21)00281-X

[18] Timmerman, R.A. (2007) A Mobility Protocol for Critically Ill Adults. Dimensions of Critical Care Nursing, 26, 175-179. https://doi.org/10.1097/01.DCC.0000286816.40570.da

[19] Lad, H., Saumur, T.M., Herridge, M.S., Dos Santos, C.C., Mathur, S., Batt, J. and Gilbert, P.M. (2020) Intensive Care Unit-Acquired Weakness: Not Just another Muscle Atrophying Condition. International Journal of Molecular Sciences, 21, Article No. 7840. https://doi.org/10.3390/ijms21217840

[20] Ministry of Health, Labour and Welfare (2018) Outline of the Revision of Medical Fees for FY 2018.

https://www.mhlw.go.jp/file/05-Shingikai-10801000-Iseikyoku-Soumuka/00002071 12.pdf

[21] The Japanese Society of Intensive Care Medicine Early Rehabilitation Study Committee (2017) Evidence Based Expert Consensus for Early Rehabilitation in the Intensive Care Unit. Journal of the Japanese Society of Intensive Care Medicine, 24, 255-303. https://doi.org/10.3918/jsicm.24_255

[22] Saitou, D. (2016) Early Rehabilitation in the Intensive Care Unit. Journal of the Kyorin Medical Society, 47, 37-41.

[23] Sato, S. and Kozaki, T. (2015) Outcome of the Introduction of the Rehabilitation System in the Emergency Center Comparison of the Number of Days It Takes to Start Rehabilitation. In: Nihon Kango Gakkai ronbunshu. Kyuseiki kango, publisher, 234-237.

[24] Oda, E. (2015) Effect of Using the Micro Vibration Therapy and Balance Ball Exercise as a Method to Increase the Range of Motion of Joints. Juushou shinshin shougai no ryouiku, 10, 123.

[25] Kimura, N. (2016) Reducing Pain and Discomfort during Care Due to Joint Contractures: Continuing the Effects of Micro-Vibration Therapy. Journal of the Japanese Association of Rural Medicine, 65, 584.

[26] De Domenico, G., Wood, E.C. and Beard, G.I. (2003) Beard's Massage. Enterprise, Tokyo.

[27] Azuma, M., Imura, Y. and Akazawa, C. (2021) Visualization of Hand Techniques in Manual Micro-Vibration Therapy. Journal of the Medical Association of Osaka Medical and Pharmaceutical University, 80, 61-69.

[28] Nakamura, M., Sato, S., Kiyono, R., and Yawata, K. (2020) Effect of Stretching on Muscle Hardness (Stiffness/Elastic Modulus) (Special Feature: Muscle Hardness and Softness). Journal of Training Science for Exercise and Sport, 32, 203-208.

[29] Nakamura, M., lkezoe, T., Takeno, Y. and Otsuka, N. (2013) Relationship between Muscle Stiffness Measured by the Muscle Hardness Meter and Passive Torque or Myotendinous Junction Displacement. Journal of the Japanese Physical Therapy Association, 40, 193-199.

[30] Akai, M. (2003) Joint Contracture: Its Prevention and Treatment. The Japanese Journal of Rehabilitation Medicine, 40, 76-80.

https://doi.org/10.2490/jirm1963.40.76 
[31] Ueda, S. and Okawa, Y. (1995) Disuse Syndrome and Physical Therapy: The Position of Disuse Syndrome and Overuse and Misuse Symptoms in Rehabilitation Medicine. The Japanese Journal of Physical Therapy, 29, 824-833.

[32] Koeda, T., Omura, T. and Okimura, K. (2019) The Treatment Time and Duration of Effectiveness of Massage Therapy. Journal of Musculoskeletal Pain Research, 11, 122-128.

[33] Koeda, T. and Amano, Y. (2010) Measurement of Muscle Hardness Using a Hardness Meter: Reproducibility, Validity and Usefulness. The Nagoya Gakuin Daigaku Ronshu; Journal of Nagoya Gakuin University; Humanities and Natural Sciences, 46, 55-61.

[34] Oroszi, T., van Heuvelen, M.J., Nyakas, C. and van der Zee, E.A. (2020) Vibration Detection: Its Function and Recent Advances in Medical Applications. F1000Res, 9. https://doi.org/10.12688/f1000research.22649.1

[35] Alam, M.M., Khan, A.A. and Farooq, M. (2018) Effect of Whole-Body Vibration on Neuromuscular Performance: A Literature Review. Work, 59, 571-583. https://doi.org/10.3233/WOR-182699

[36] Alghadir, A.H., Anwer, S., Zafar, H. and Iqbal, Z.A. (2018) Effect of Localised Vibration on Muscle Strength in Healthy Adults: A Systematic Review. Physiotherapy, 104, 18-24. https://doi.org/10.1016/j.physio.2017.06.006

[37] Rauch, F. (2009) Vibration Therapy. Developmental Medicine \& Child Neurology, 51, 166-168. https://doi.org/10.1111/j.1469-8749.2009.03418.x

[38] Fowler, B.D., Palombo, K.T.M., Feland, J.B. and Blotter, J.D. (2019) Effects of WholeBody Vibration on Flexibility and Stiffness: A Literature Review. International Journal of Exercise Science, 12, 735-747.

[39] Homoma, H., Saito, K. and Takahashi, T. (2021) Effect of Titubation as a Stimulus on Body Flexibility. Rigakuryoho Kagaku, 36, 107-112. https://doi.org/10.1589/rika.36.107

[40] Nakagami, G., Urasaki, M., Kitagawa, A. and Sanada, H. (2010) Focus Translational Research in Nursing: The Process of Developing and Testing a Device to Promote the Treatment of Pressure Ulcers by Vibration. Investigation of the Effect of Vibration on Blood Flow-Experiments on Healthy Subjects. The Japanese Journal of Nursing Research, 43, 459-464.

[41] Ichioka, S., Yokogawa, H., Sekiya, N. and Nakagami, G. (2010) Translational Research in Nursing the Process of Developing and Validating a Device to Promote the Treatment of Bedsores by Vibration. Discovery of Seeds. Elucidation of Pathological Mechanism. Mechanism of Microcirculation Change by Vibration. The Japanese Journal of Nursing Research, 43, 453-458.

[42] Kanazashi, M., Fujita, N. and Fujino, H. (2013b) Preventive Effect of Combined Load-Bearing and Nutritional Support on Mitochondrial Dysfunction Associated with Disuse Muscle Atrophy. Journal of the Japanese Physical Therapy Association, 40, 125.

[43] Kanazashi, M., Fujita, N., Murakami, S. and Fujino, H. (2013) Protective Effects of Astaxanthin Supplementation on Capillary Regression of Disuse Atrophied Muscle. Japanese Journal of Physical Therapy Fundamentals, 16, 39-46.

[44] Vollman, K.M. (2013) Understanding Critically Ill Patients Hemodynamic Response to Mobilization: Using the Evidence to Make It Safe and Feasible. Critical Care Nursing Quarterly, 36, 17-27. https://doi.org/10.1097/CNQ.0b013e3182750767

[45] Convertino, V.A., Doerr, D.F., Eckberg, D.L., Fritsch, J.M. and Vernikos-Danellis, J. (1990) Head-Down Bed Rest Impairs Vagal Baroreflex Responses and Provokes 
Orthostatic Hypotension. Journal of Applied Physiology, 68, 1458-1464. https://doi.org/10.1152/jappl.1990.68.4.1458

[46] Kimura, T., Tsuda Y., Uchida S. and Eboshida, A. (2006) Association of Perceived Stress and Stiff Neck/Shoulder with Health Status: Multiple Regression Models by Gender. Hiroshima Journal of Medical Sciences, 55, 101-107.

[47] Okuno, H., Takeda, T., Sasaoka, T. and Fukuda, F. (2009) Relationship between Katakori (Shoulder Stiffness) and Shoulder Hardness. Journal of the Japan Society of Acupuncture and Moxibustion, 59, 30-38.

[48] Fujii, T., Matsudaira, K., Noma, K., Ishizuka, A. and Yamada, K. (2012) Objective Measurement of Neck-Shoulder Discomfort and Analysis of Associated Factors. Clinical Orthopaedic Surgery, 47, 619-624.

[49] Yabuki, S. (2007) Pathogenesis of the Neck-Shoulder Stiffness (Katakori). Clinical Orthopaedic Surgery, 42, 413-417. https://doi.org/10.3777/jisam.59.30 